\title{
44448 - EFFECTS OF ACTIVATED PROTEIN C IN THE RAT MESENTERIC MICROCIRCULATION
}

Disclosure: Grants/research support - Activated protein $\mathrm{C}$ was provided by the Eli Lilly.

\author{
Christian Lehmann, Department of Anesthesia, Dalhousie University, Halifax, NS, \\ Canada; \\ Ricardo Scheibe, Department of Anesthesia, Ernst-Moritz-Arndt-University; \\ Michael Schade, Department of Anesthesia, Ernst-Moritz-Arndt-University; \\ TI Usichenko, Department of Anesthesia, Ernst-Moritz-Arndt-University; \\ K Meissner, Department of Anesthesia, Ernst-Moritz-Arndt-University; \\ M Gründling, Department of Anesthesia, Ernst-Moritz-Arndt-University; \\ $M$ Wendt, Department of Anesthesia, Ernst-Moritz-Arndt-University; \\ D Pavlovic, Department of Anesthesia, Ernst-Moritz-Arndt-University;
}

INTRODUCTION: The large PROWESS trial showed the efficacy of recombinant human activated protein $\mathrm{C}$ (aPC) in severe sepsis (1). However, the mechanisms of action of the aPC in severe sepsis are not completely elucidated. Therefore, it would be important to examine the effects of aPC on various mechanisms that are believed to be playing a role in severe sepsis with the scope to define the mechanisms responsible for its probable efficacy and thereby open new therapeutic possibilities for sepsis. The aim of our study was to evaluate the effects of aPC on the mesenteric microcirculation (leukocyte-endothelial interaction, plasma extravasation) during experimental endotoxemia in rats.

METHODS: After approval by the local standing committee on animal experiments we performed a prospective, randomized, controlled animal study in 40 male Lewis rats. Group 1 (control; $n=10$ ) served as healthy control group. Group 2 (LPS; $n=10)$ and group 4 (LPS+aPC; $\mathrm{n}=10$ ) received an endotoxin bolus i.v. (15 mg/kg lipopolysaccharide, LPS). In group 3 (aPC; $\mathrm{n}=10$ ) and group 4 (LPS+aPC) $2 \mathrm{mg} / \mathrm{kg}$ aPC were administered before endotoxin or placebo. Leukocyte-endothelial interactions and mesenteric plasma extravasation were determined at 0,1 and 2 hours during the experiment by intravital fluorescence microscopy (IVM). For this purpose, leukocytes were stained with intravenously administered Rhodamine 6G and plasma stream was marked with FITC-albumine.

RESULTS: During endotoxemia, a significant increase of leukocyte rolling behaviour, leukocyte adherence (see figure) and plasma extravasation in the LPS group was observed. APC treatment reduced leukocyte adherence (fig.) and plasma extravasation ( $-28.8 \%$ vs. LPS group, $\mathrm{p}<.05)$ during endotoxemia significantly compared to untreated LPS animals. Also in group 3 (aPC) a significant decrease of leukocyte adherence (fig.) and mesenteric plasma extravasation (-17.7 \% vs. control group, $\mathrm{p}<.05$ ) was to be seen.

DICUSSION: In this study, aPC treatment attenuated significantly the detrimental impact of endotoxemia on parameters of the mesenteric microcirculation in rats. Because of the crucial role of the microcirculation in the pathogenesis of ongoing sepsis and multiple organ dysfunction syndrome, these effects may be of clinical importance $(2,3)$. 
REFERENCES: (1) Bernard GR et al. 2001. Efficacy and safety of recombinant human activated protein C for severe sepsis. N Engl J Med. 344:699-709. (2) Spronk PE et al. 2004. Bench-tobedside review: Sepsis is a disease of the microcirculation. Critical Care 8:462-8. (3) Iba T et al. 2005. Activated protein $\mathrm{C}$ improves the visceral microcirculation by attenuating the leukocyteendothelial interaction in a rat lipopolysaccharide model. Crit Care Med. 33:368-72.

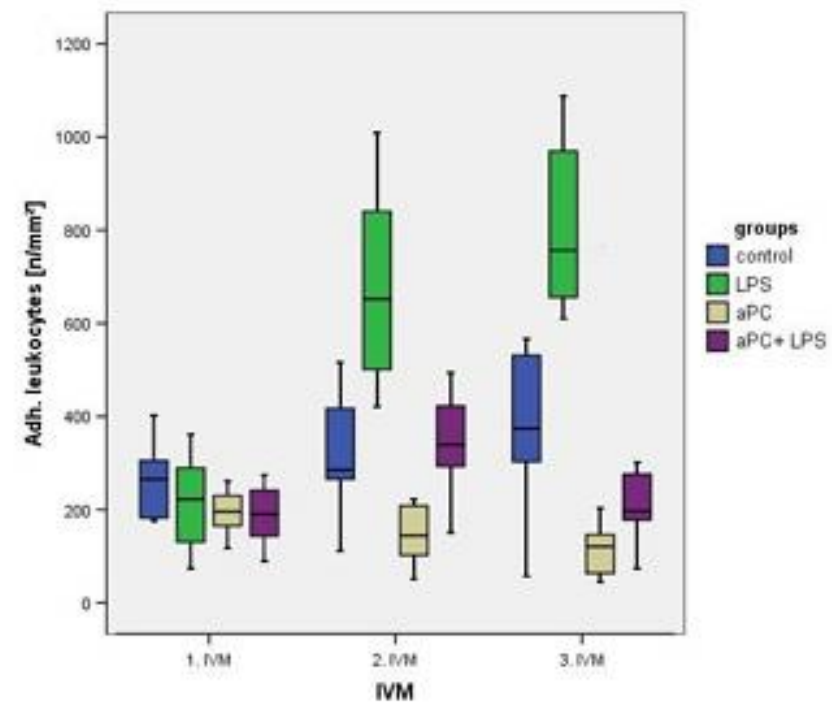

